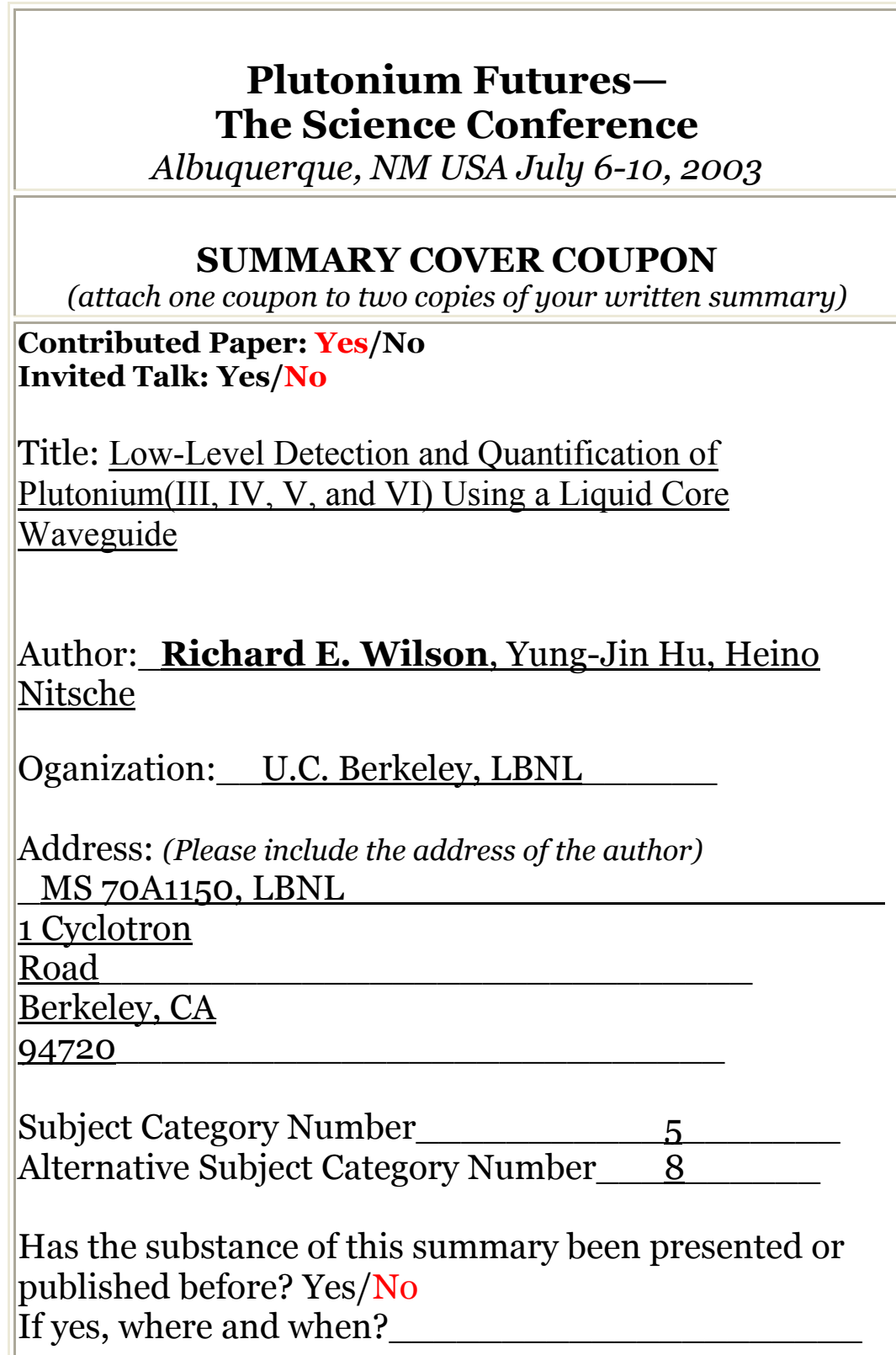

Has the paper been submitted for publication in a technical journal? Yes/No

If yes, when and what publication?

Has this summary been approved for publication by your institution or company? Yes/No

Plutonium Futures - The Science Conference is unclassified and open to the general public. It is your responsibility to protect classified, sensitive, or proprietary information. 


\title{
Low-Level Detection and Quantification of Plutonium(III, IV, V, and VI) Using a Liquid Core Waveguide
}

\author{
Richard E. Wilson ${ }^{1,2}$, Yung-Jin $\mathrm{Hu}^{1,3}$, Heino Nitsche ${ }^{1,3}$
}

1. Department of Chemistry, University of California, Berkeley. Berkeley, California.

2. Chemical Sciences Division, Glenn T. Seaborg Center, Lawrence Berkeley National Laboratory. Berkeley, California.

3. Nuclear Sciences Division, Lawrence Berkeley National Laboratory. Berkeley, California.

Understanding the aqueous chemistry of plutonium, in particular in environmental conditions, is often complicated by plutonium's complex redox chemistry. Because plutonium possesses four oxidation states, all of which can coexist in solution ${ }^{1}$, a reliable method for the identification of these oxidation states is needed.

The identification of plutonium oxidation states at low levels in aqueous solution is often accomplished through an indirect determination using series of liquid-liquid extraction procedures using oxidation state specific reagents such as HDEHP and TTA ${ }^{2}$. While these methods, coupled with radioactive counting techniques provide superior limits of detection they may influence the plutonium redox equilibrium, are time consuming, waste intensive and costly. Other analytical methods such as mass spectrometry and radioactive counting as stand alone methods provide excellent detection limits but lack the ability to discriminate between the oxidation states of the plutonium ions in solution.

Traditionally, UV-Vis absorption spectroscopy has been used to identify oxidation state purity or mixtures in concentrated plutonium solutions. However, under the conditions of environmental studies the plutonium concentration is often $10^{-6} \mathrm{M}$ or 
below. The small molar absorpitivity of the plutonium ions ${ }^{3}$ make detection by optical absorption spectroscopy at these levels impossible. To improve detection, laser photoacoustic spectroscopy has been applied to plutonium solutions with certain oxidation states in near environmental concentrations.

To overcome the high detection limits associated with plutonium ions in solution, a one-meter liquid core waveguide was employed. The waveguide, consisting of a Teflon capillary with a fill volume of 250 microliters and an effective pathlength of one-meter should provide an enhancement in detection due to the one hundred-fold increase in pathlength versus a conventional one centimeter cell. This method has the advantage of non-destructively sampling the plutonium solution and also does not generate the mixed waste that is a product of the extraction protocols.

The results of this study showed that the actual increase in the detection limits were between 18 and 33 times that of the one centimeter cell (Table 1). Calculation of the molar absorptivities from standard plots (Table 2) showed excellent agreement with literature values ${ }^{3,4}$. Limits of detection for the waveguide were calculated to be $16 \times 10^{-6}$ $\mathrm{M}$ for $\mathrm{Pu}(\mathrm{V}), 8 \times 10^{-6} \mathrm{M}$ for $\mathrm{Pu}(\mathrm{III}), 5 \times 10^{-6} \mathrm{M}$ for $\mathrm{Pu}(\mathrm{IV})$, and $7 \times 10^{-7}$ for $\mathrm{Pu}(\mathrm{VI})$.

These detection limits allow for the identification of plutonium oxidation states in solution at concentrations relevant to selected laboratory scale environmental studies. The fill volume of the one-meter waveguide is 250 microliters, and rapid filling and purging can be accomplished using a small peristaltic pump.

This method is currently being used in the determination of plutonium oxidation states in the supernatant of plutonium sorption experiments with iron and manganese oxides. The ability to identify oxidation states in solution in these experiments has 
yielded important information regarding the redox processes that occur in solution and at the mineral interface.

Minimal sampling time, small volumes of waste, and the enhanced detection limits compared to those of traditional spectrophotometry make this a viable method for the identification and quantification of plutonium oxidation states in solution.

Experiments are currently underway to qualify longer pathlength cells of 5 and 10 meters to further enhance the detection capabilities of these systems.

Table 1. Calculated molar absorptivities and detection limits using a one-meter waveguide. Values in parentheses are taken from reference 3.

\begin{tabular}{cccc}
\hline Pu Ion & Absorption Wavelength $(\mathrm{nm})$ & Extinction Coefficient $\left(\mathrm{M}^{-1} \mathrm{~cm}^{-1}\right)$ & Limit of Detection $(\mu \mathrm{M})$ \\
\hline III & 600 & $33.8(38)$ & 7.97 \\
IV & 470 & $49.0(55)$ & 5.32 \\
V & 568 & $18.8(19)$ & 15.7 \\
VI & 830 & $287.9(550)$ & 0.71 \\
\hline
\end{tabular}

\section{References}

1. Cleveland, J.M., The Chemistry of Plutonium. 1970, New York, Gordon and Breach Scientific Publishers.

2. Nitsche, H., Lee, S., Gatti, R., Determination of Plutonium Oxidation States at Trace Levels Pertinent to Nuclear Waste Disposal. Journal of Radioanalytical and Nuclear Chemistry-Articles, 1988. 124(1): p. 171-185. 
3. Cohen, D.J., The Absorption Spectra of Plutonium Ions in Perchloric Acid

Solution. Journal of Inorganic and Nuclear Chemistry, 1961. vol. 18. p. 211218.

4. Silva, R.J., Nitsche, H., Actinide Environmental Chemistry. Radiochimica Acta, 1995, vol. 70-71, p. 377-396. 